\title{
Magnetism, exchange and crystal field parameters in the orbitally unquenched Ising antiferromagnet $\mathrm{FePS}_{3}$
}

\author{
NIRMALA CHANDRASEKHARAN and SUKUMARAN VASUDEVAN \\ Department of Inorganic and Physical Chemistry, Indian Institute of Science, Bangalore \\ 560012 , India
}

MS received 7 January 1994

\begin{abstract}
FePS}_{3}$ is a layered antiferromagnet $\left(T_{\mathrm{N}}=123 \mathrm{~K}\right)$ with a marked Ising anisotropy in magnetic properties. The anisotropy arises from the combined effect of the trigonal distortion from octahedral symmetry and spin-orbit coupling on the orbitally degenerate ${ }^{5} T_{2 g}$ ground state of the $\mathrm{Fe}^{2+}$ ion. The anisotropic paramagnetic susceptibilities are interpreted in terms of the zero field Hamiltonian, $\mathscr{H}=\Sigma_{i}\left[\Delta\left(L_{i z}^{2}-2\right)+|\lambda| \mathbf{L}_{i} \cdot \mathbf{S}_{i}\right]-\Sigma_{i j} J_{i j} \mathbf{S}_{i} \cdot \mathbf{S}_{j}$. The crystal field trigonal distortion parameter $\Delta$, the spin-orbit coupling $\lambda$ and the isotropic Heisenberg exchange, $J_{i j}$, were evaluated from an analysis of the high temperature paramagnetic susceptibility data using the Correlated Effective Field (CEF) theory for many-body magnetism developed by Lines. Good agreement with experiment were obtained for $\Delta / k=215.5 \mathrm{~K}$; $\lambda / k=166.5 \mathrm{~K} ; J_{n n} / k=27.7 \mathrm{~K}$; and $J_{n n n} / k=-2.3 \mathrm{~K}$. Using these values of the crystal field and exchange parameters the CEF predicts a $T_{\mathrm{N}}=122 \mathrm{~K}$ for FePS ${ }_{3}$, which is remarkably close to the observed value of the $T_{\mathrm{N}}$. The accuracy of the CEF approximation was also ascertained by comparing the calculated susceptibilities in the CEF with the experimental susceptibility for the isotropic Heisenberg layered antiferromagnet $\mathrm{MnPS}_{3}$, for which the high temperature series expansion susceptibility is available.
\end{abstract}

Keywords. Layered antiferromagnet; crystal field; anisotropy; correlated effective field.

PACS Nos 75.10; 75·30; 75·70

\section{Introduction}

The layered transition metal thiophosphates $M \mathrm{PS}_{3}(M=\mathrm{Mn}, \mathrm{Fe}, \mathrm{Ni})$ are a unique class of compounds; they represent one of the few known layered systems in which both magnetic and crystallographic lattices are two-dimensional $[1,2]$. Unlike most other $2 D$ magnetic systems e.g. those belonging to the $\mathrm{K}_{2} \mathrm{NiF}_{4}$ family [3] wherein magnetic layers are separated by diamagnetic layers, in the transition metal thiophosphates, the $M \mathrm{PS}_{3}$ layers are separated by a van der Waals gap.

The transition metal thiophosphates are insulating antiferromagnets $[4,5]$. The nature of metal-ligand interactions are ionic [6] and the $d$ electron manifold of the transition metal ion is reasonably well described in the weak field limit of crystal field theory [7]. The anisotropic magnetic susceptibility of these compounds have been reported [5]. The most remarkable feature of magnetism in these compounds is that the anisotropy of the paramagnetic susceptibility is strongly dependent on the metal ion. While the susceptibility of $\mathrm{MnPS}_{3}$ is isotropic and that of $\mathrm{NiPS}_{3}$ shows only a weak anisotropy, $\mathrm{FePS}_{3}$ exhibits a marked Ising like anisotropy. The anisotropy in these compounds has been shown to arise from crystal field effects-a combination 


\section{Nirmala Chandrasekharan and Sukumaran Vasudevan}

of spin-orbit coupling and trigonal distortion of the $M S_{6}$ octahedra, giving rise to a zero-field splitting of the ground state of the transition metal ion.

An interesting consequence of the layered structure of these compounds-the fact that the $M \mathrm{PS}_{3}$ layers are separated by a van der Waals gap-is that it is possible to intercalate a wide variety of guest molecules and ions, similar to that observed in the transition metal dichalcogenides [8]. Subsequent to intercalation the major changes and in many cases the only change, are in the magnetic properties. For example, pyridine intercalation in the isotropic Heisenberg antiferromagnet, $\mathrm{MnPS}_{3}$, leads to a weak ferromagnetic state [9] which has been shown to arise from a combination of Dzyaloshinsky - Moriya interactions and single ion anisotropy terms, neither of which were present in the parent compound [10]. Similarly, on pyridine intercalation in the Ising like antiferromagnet $\mathrm{FePS}_{3}$, a spontaneously magnetized state is realized [11], while on amine intercalation the changes have been ascribed to random field Ising model behaviour [12].

An obvious prerequisite to a better understanding of the changes in magnetic properties on intercalation, is a quantitative evaluation of the exchange and crystal field parameters of the host. In this paper we have attempted to do so for FePS from an analysis of the high temperature susceptibility data of Joy et al [5]. The model used for the analysis must in principle be able to account (i) for the complicated level structure of the transition metal ion arising from a combination of spin-orbit coupling, trigonal distortion and Zeeman splitting, which in the present case, completely lifts the 15-fold degeneracy of the ${ }^{5} T_{2 g}$ state of the $\mathrm{Fe}^{2+}$ ion and (ii) at the same time also be able to account for spin correlations which in these lowdimensional systems manifest at temperatures much higher than $T_{\mathrm{N}}$ [3]. It is for the latter reason that the mean field (MF) is a poor approximation, since by replacing all ions except one by their ensemble average, interion spin and orbital correlations are completely ignored. At the same time the complicated level structure of the $\mathrm{M}^{2+}$ ion makes it difficult to apply more refined theories, such as the renormalization group or even the high temperature series expansion (HTSE). In this paper we have used the correlated effective field (CEF) approximation developed by Lines $[13,14]$. This model is ideally suited for systems where excited orbital crystal field energies, exchange energies and thermal energies are all of the same order of magnitude. The formalism attempts to reduce the many-body problem to a single body non-interacting ensemble form, by the introduction of static temperature dependent spin correlation parameters which are evaluated by forcing consistency with the fluctuation-dissipation theorem. The model has been used with reasonable success in analyzing the high temperature susceptibility of $\mathrm{RbFeBr}_{3}$ [15] and $\mathrm{RbFeCl}_{3}$ [16].

In $\S 2$ we provide a brief outline of the CEF formalism. In $\$ 3$ we discuss the CEF theory in the simpler context of a $2 D$ isotropic Heisenberg system, by comparing the calculated susceptibilities in the CEF approximation with the experimental data for $\mathrm{MnPS}_{3}$ and also the exact HTSE fit for the same. This was done to estimate the accuracy of the CEF approximation. The results are also compared with the analysis from other approximations such as the Oguchi $[17,18]$ and the MF treatment. In $\S 4$ the crystal field levels of $\mathrm{Fe}^{2+}$ in the trigonally distorted environment of $\mathrm{FePS}_{3}$ are described and the CEF calculations for $\mathrm{FePS}_{3}$ performed. The exchange and crystal field parameters were evaluated by fitting the CEF susceptibilities to the experimental data. Finally in $\S 5$ we summarize the results. 


\section{Anisotropic magnetic susceptibility}

\section{The correlated effective field approximation $[13,14]$}

The CEF approximation is applicable for systems where the total spin Hamiltonian may be written as

$$
\mathscr{H}=\mathscr{H}_{\mathrm{CF}}+\sum_{i j} J_{i j} \mathbf{S}_{i} \mathbf{S}_{j}
$$

where $\mathscr{H}_{\mathrm{CF}}$ is the Hamiltonian in the absence of exchange.

In this model, the CEF for the $i$ th spin $S_{i}$ is obtained by replacing each $S_{j}$ in (1) by the sum of two contributions, one its ensemble average $\left\langle S_{j}\right\rangle$, the other, a term proportional to the instantaneous deviation of $S_{i}$ from its ensemble average value $\left\langle S_{i}\right\rangle$ i.e.

$$
S_{j}^{\lambda} \rightarrow\left\langle S_{j}^{\lambda}\right\rangle+\alpha^{\lambda}\left(S_{i}^{\lambda}-\left\langle S_{i}^{\lambda}\right\rangle\right), \quad \lambda=x, y, z .
$$

The $\alpha^{\lambda}$ 's are temperature dependent static correlation parameters. Corresponding to this replacement, the effective Hamiltonian for the $i$ th spin in the high temperature paramagnetic phase, where ensemble averages are zero, is

$$
\mathscr{H}_{i}^{0}(\mathrm{eff})=\mathscr{H}_{\text {CF }}-\sum_{j \lambda} 2 J_{i j}^{\lambda} \alpha^{\lambda}\left(S_{i}^{\lambda}\right)^{2}
$$

In the presence of a field $h=g \beta H^{0}$ applied in a direction $\gamma$, the effective Hamiltonian becomes

$$
\mathscr{H}_{i}(\mathrm{eff})=\mathscr{H}_{i}^{0}(\mathrm{eff})-h \mu^{\gamma}+2 \sum_{j \gamma} J_{i j}^{\gamma} S_{i}^{\gamma}\left(\left\langle S_{j}^{\gamma}\right\rangle-\alpha^{\gamma}\left\langle S_{i}^{\gamma}\right\rangle\right)
$$

where $\mu=2 S+L$. The ensemble averages in direction $\lambda \neq \gamma$ are zero.

It may be seen that $\mathscr{H}_{i}(\mathrm{ef})$ approximates the many-body Hamiltonian, by a single particle one and except for the sign of $J$, is identical for the ferromagnetic and antiferromagnetic case.

The field dependent ensemble averages are obtained by treating the last two terms of (4) as a perturbation on $\mathscr{H}_{i}^{0}$ (eff). $\mathscr{H}_{i}^{0}$ (eff) may be diagonalized and the eigenfunctions $\left|\psi_{n, \alpha}\right\rangle$ and eigenvalues $E_{n, \alpha}$ obtained as a function of the correlation parameters $\alpha$. The field dependent ensemble averages are

$$
\begin{aligned}
& k T\left\langle S(q)^{\gamma}\right\rangle_{h}=h(q)\left\langle\mu_{i}^{\gamma}: S_{i}^{\gamma}\right\rangle_{0}+2\left[J(q)^{\gamma}-\alpha^{\gamma} J^{\gamma}(0)\right]\left\langle S(q)^{\gamma}\right\rangle_{h}\left\langle S_{i}^{\gamma}: S_{i}^{\gamma}\right\rangle_{0} \\
& k T\left\langle L(q)^{\gamma}\right\rangle_{h}=h(q)\left\langle\mu_{i}^{\gamma}: L_{i}^{\gamma}\right\rangle_{0}+2\left[J(q)^{\gamma}-\alpha^{\gamma} J^{\gamma}(0)\right]\left\langle S(q)^{\gamma}\right\rangle_{h}\left\langle S_{i}^{\gamma}: S_{i}^{\gamma}\right\rangle_{0}
\end{aligned}
$$

where $S(q)$ and $L(q)$ are the fourier transform of the corresponding lattice quantity and $J(q)=\Sigma_{j-i} J_{i j} \exp \left(i q r_{i j}\right)$ where $r_{i j}$ is the vector connecting the ith atom to its $j$ th neighbour and momentum $q$ belongs to the first Brillouin zone. The colon product ensemble average is defined by

$$
\langle A: B\rangle=\sum_{n} \rho_{n}\left(A_{n n} B_{n n}+2 k T \sum_{m, \alpha \neq n, \alpha} \frac{A_{n m} B_{m n}}{E_{m, \alpha}-E_{n, \alpha}}\right),
$$




\section{Nirmala Chandrasekharan and Sukumaran Vasudevan}

where $A_{n m}=\langle n, \alpha|A| m, \alpha\rangle . \rho_{n}$ is the density matrix given by

$$
\rho_{n}=\frac{\exp \left(-E_{n, \alpha} / k T\right)}{\sum_{n} \exp \left(-E_{n, \alpha} / k T\right)}
$$

The wave vector dependent susceptibility is then

$$
\chi^{\gamma}(q)=\left\langle\mu^{\gamma}(q)\right\rangle / h(q)=\frac{\left\langle 2 S^{\gamma}(q)+L^{\gamma}(q)\right\rangle_{h}}{h(q)} .
$$

Substituting for the ensemble averages from (5) and (6)

where

$$
k T \chi_{i}(q)=\left\langle\mu_{i}^{\gamma}: \mu_{i}^{\gamma}\right\rangle_{0}+K(q),
$$

$$
K(q)=\frac{2\left[J(q)^{\gamma}-\alpha^{\gamma} J^{\gamma}(0)\right]\left\langle\mu_{i}^{\gamma}: S_{i}^{\gamma}\right\rangle_{0}^{2}}{k T-2\left[J(q)^{\gamma}-\alpha^{\gamma} J^{\gamma}(0)\right]\left\langle S_{i}^{\gamma}: S_{i}^{\gamma}\right\rangle_{0}} .
$$

From the fluctuation theorem we have [13] $\Sigma_{q} K(q)=0$ which allows for the complete determination of the correlation parameter $\alpha$

$$
\alpha^{\lambda}=\frac{\sum_{q} J(q)\left[k T-2\left(J(q)-\alpha^{\lambda} J(0)\right)\left\langle S_{l}^{\lambda}: S_{i}^{\lambda}\right\rangle_{0}\right]^{-1}}{\sum_{q} J(0)\left[k T-2\left(J(q)-\alpha^{\lambda} J(0)\right)\left\langle S_{i}^{\lambda}: S_{i}^{\lambda}\right\rangle_{0}\right]^{-1}} .
$$

Once $\alpha^{\alpha}$ is obtained, the uniform static susceptibility directly follows

$$
k T \chi_{0}^{\gamma}=\left\langle\mu_{i}^{\gamma}: \mu_{i}^{\gamma}\right\rangle_{0}+\frac{\left\langle\mu_{i}^{\gamma}: S_{i}^{\gamma}\right\rangle_{0}^{2} 2 \sum_{j} J_{i j}(0)\left(1-\alpha^{\gamma}\right)}{k T-\left\langle S_{i}^{\gamma}: S_{i}^{\gamma}\right\rangle_{0} 2 z \sum_{j} J_{i j}(0)\left(1-\alpha^{\gamma}\right)} .
$$

The above formalism is valid without restriction on the sign of $J$. For an antiferromagnet the Neel temperature - the temperature at which the staggered susceptibility, $\chi^{\lambda}\left(q=q^{*}\right)$ diverges ( $q^{*}$ is the antiferromagnetic ordering vector) - may be calculated in the CEF model as the temperature at which the denominator of (10) goes to zero (for $q=q^{*}$ ). Noting that for an antiferromagnet $J(q)=-J(0)$ it follows that the Neel temperature, $T_{\mathrm{N}}$ is

$$
k T_{N}^{\lambda}=2 J(0) z\left(1+\alpha^{\lambda}\right)\left\langle S_{i}^{\lambda}: S_{i}^{\lambda}\right\rangle_{0} .
$$

It may be seen that the calculation of $\alpha$ 's are crucial to the evaluation of susceptibilities as well as $T_{\mathrm{N}}$. The calculation of $\alpha$ 's involves the summation over the Brillouin zone. We have used the special $k$ point scheme introduced by Chadi and Cohen [19] for obtaining the averages over the Brillouin zone. For $\mathrm{MnPS}_{3}$ and $\mathrm{FePS}_{3}$ for which the in-plane structure is the non-primitive honeycomb lattice, the special $k$ points for the $2 D$ hexagonal lattice reported by Cunningham were employed [20]. 


\section{Comparison with a $2 D$ isotropic Heisenberg antiferromagnet, $\mathrm{MnPS}_{3}$}

In order to gauge the strength and weakness as well as the accuracy of the CEF approximation, it is necessary to compare with the results of a more accurate or exact calculation. For this reason the calculated susceptibilities in the CEF approximation have been compared with the experimental data for the $2 D$ isostructural $S=5 / 2$ Heisenberg antiferromagnet, $\mathrm{MnPS}_{3}$, for which the exact high temperature series expansion susceptibility is known. This exercise was also necessary to test the efficacy of the special $k$ point scheme for providing the Brillouin zone average for a honeycomb lattice in the calculation of $\alpha$, the static correlation parameter.

The results of the comparison are shown in figure 1 . The experimental susceptibilities are from ref. [5]. The HTSE expression for the susceptibility of a $2 D$ Heisenberg antiferromagnet as

$$
\chi_{m}=\frac{N g^{2} \beta^{2} S(S+1)}{3 k T} \cdot \frac{1}{1+\sum_{i=1}^{6}(-1)^{i} b_{i}(|J| / k T)^{i}}
$$

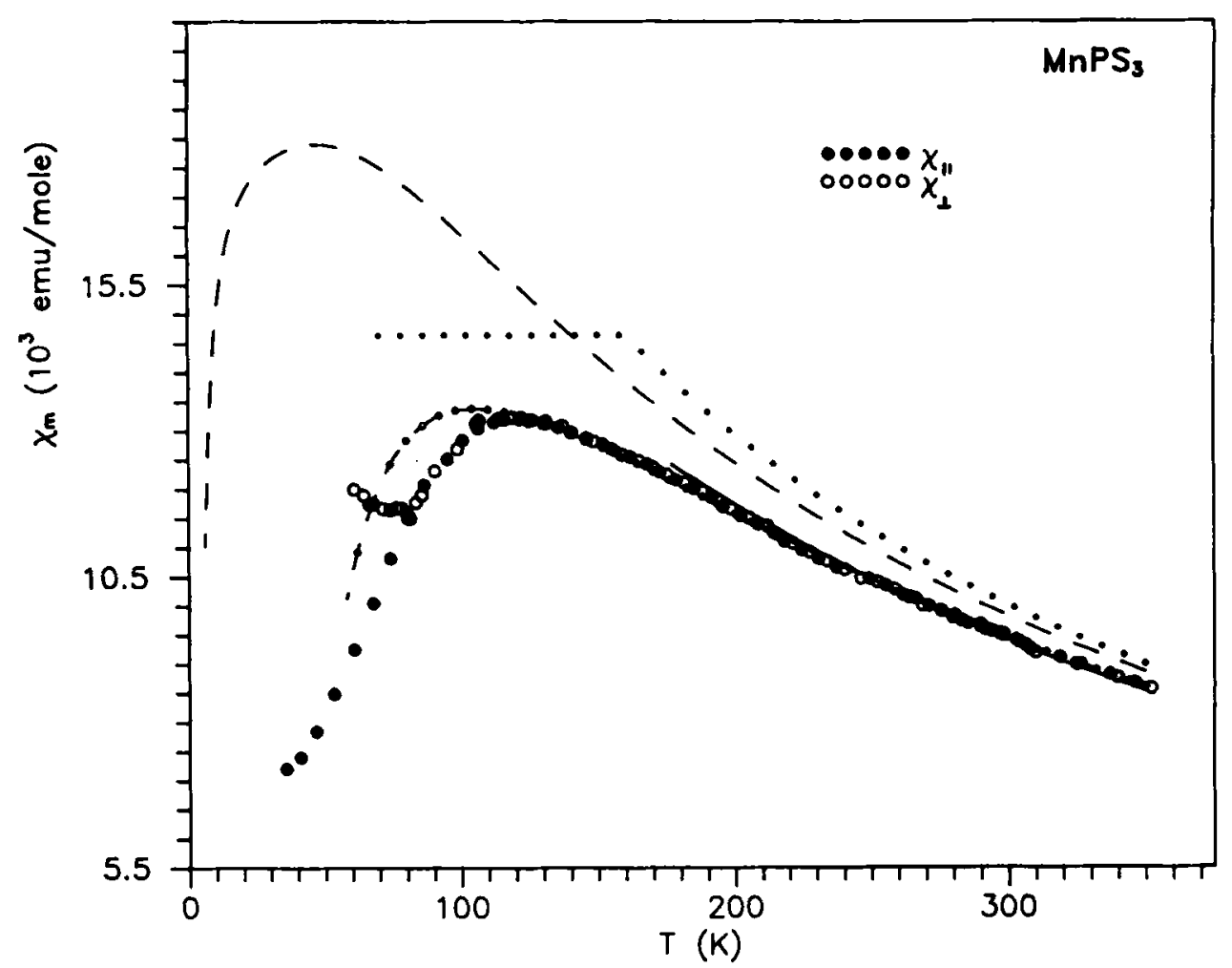

Figure 1. The isotropic magnetic susceptibilities, $\chi_{1}$ and $\chi_{1}$ for $\mathrm{MnPS}_{3}$ as a function of temperature. $\chi_{\|}$and $\chi_{\perp}$ refer to the direction of the applied field with respect to the trigonal axis. $\left(-\cdot \cdot_{-} \cdot{ }^{-}-\right)$is the fit to the HTSE susceptibility expression in (12) for $J / k=-8 \cdot 1 \mathrm{~K}$ and $g=2 \cdot 01$. ( - ) is the fit of the calculated susceptibility in the CEF approximation for the same values of the parameters as in HTSE, while the (-- ) and (....) lines are the calculated susceptibilities in the Oguchi and MFA for the same value of $J / k$ and $g$. 


\section{Nirmala Chandrasekharan and Sukumaran Vasudevan}

The $b_{i}$ 's are the expansion coefficients, and depend on the type of magnetic lattice. The $(-1)^{i}$ term, accounts for the antiferromagnetic nature of exchange in the system. For a honeycomb lattice, to which $\mathrm{MnPS}_{3}$ belongs, the constants have been calculated using the formula of Rushbrook and Wood [21]. The dashed-dotted line in figure 1 is the fit for the HTSE susceptibility. The best fit is obtained for $J=-8.1 \mathrm{~K} ; g=2.01$.

The solid line in figure 1 is the CEF susceptibility for $\mathrm{MnPS}_{3}$ calculated using the same value of $J$ and $g$ which gave the best fit for the HTSE. The calculation of the CEF susceptibility for $\mathrm{MnPS}_{3}$ is straightforward. $\mathscr{H}_{C F}$ is zero in (4) and $\alpha$ was evaluated from (9) using the special $k$ point summation for the Brillouin zone averages [20].

It may be seen that the CEF susceptibility compares extremely well with the exact HTSE susceptibility. (The CEF susceptibility is not calculable above $177 \mathrm{~K}$ since the denominator in (10) goes to zero. This temperature however has no physical significance since in these $2 D$ Heisenberg antiferromagnets the actual $T_{\mathrm{N}}$ is a consequence of weak $3 D$ interlayer coupling).

It is also interesting to compare the results of the CEF approximation with other approximations such as the Oguchi molecular field $[17,18]$ which also introduce an element of spin correlation while retaining the simplicity of the mean field. In the Oguchi approximation [18] a small section of the crystal e.g. a pair of ions is treated exactly while interactions with the rest of the ions in the crystal are approximated by an effective field.

The susceptibility of $\mathrm{MnPS}_{3}$ in the Oguchi approximation was derived from the exact expression for $\left\langle S^{\lambda}\right\rangle_{\text {dimer }}$ for a pair of $\mathrm{Mn}^{2+}$ spins $\left(s_{1}=s_{2}=5 / 2\right)$. The susceptibility is

$$
\begin{aligned}
\chi_{m} & =\frac{N g^{2} \beta^{2}}{k T} \cdot \frac{F(J, T)}{G(J, T)-2(z-1) j F(J, T)} ; j=J / k T \\
F(J, T) & =1+5 \exp (4 j)+14 \exp (10 j)+30 \exp (18 j)+55 \exp (28 j) \\
G(J, T) & =3+\exp (-2 j)+5 \exp (4 j)+7 \exp (10 j)+9 \exp (18 j)+11 \exp (28 j)
\end{aligned}
$$

Figure 1 shows the Oguchi susceptibility, calculated using $J / k=-8 \cdot 1 \mathrm{~K}$ and $g=2 \cdot 01$. These values are the ones which gave the best fit to the HTSE susceptibility.

The Oguchi susceptibility although an improvement on the simple MFA still underestimates the antiferromagnetic correlation and is a poor second to the CEF, in comparison with the HTSE results.

The results of figure 1 clearly establish the effectiveness of the CEF approximation in evaluating the high temperature susceptibility of $2 \mathrm{D}$ systems. The results also establish the effectiveness of the special $k$ point scheme in providing Brillouin zone averages in the calculation of the correlation parameter, $\alpha$, the calculation of which is crucial in obtaining the correct values of the susceptibility in the CEF model.

\section{CEF approximation applied to $\mathrm{FePS}_{3}$}

In $\mathrm{FePS}_{3}$ the $d^{6}$ free ion ground term ${ }^{5} \mathrm{D}$ is split by the the octahedral crystal field into an upper orbtial doublet and a lower orbital triplet $\left({ }^{5} T_{2}\right)$. In considering the effect of the trigonal distortion and spin-orbit coupling, the interaction of the ${ }^{5} T_{2}$ state with the higher lying excited states are ignored. This is justifiable since it is known from optical absorption data that the closest lying excited state, ${ }^{5} E$, is 


\section{Anisotropic magnetic susceptibility}

$8700 \mathrm{~cm}^{-1}$ above the ground state. Within the lower triplet, use is made of the structural isomorphism of the $T_{2}$ and $P$ symmetry groups to define a fictitious orbital angular momentum $L^{\prime}=1$ [22]. For the ${ }^{5} T_{2}$, the matrix clements of the orbital angular momentum $L$ are -1 times the equivalent elements of $L^{\prime}$ within the $P$ states; $L=\alpha L^{\prime}(\alpha=-1)$. In terms of the fictitious orbital angular momenta the Hamiltonian representing the trigonal distortion from $O_{h}$ symmetry as well as spin-orbit coupling has the form,

$$
\mathscr{H}=\Delta\left(L_{z}^{\prime 2}-2\right)-|\lambda| \mathbf{L}^{\prime} \cdot \mathbf{S}
$$

The first term represents the trigonal component perturbation; $z$ is the axis of this low symmetry perturbation. The Hamiltonian is evaulated within the 15 states, $\left|M_{S}, M_{L^{\prime}}\right\rangle$ of a ${ }^{5} P$ term. This breaks up into seven submatrices. These may be labelled by their $M_{J}$ values which in the absence of a field is a good quantum number. There is essentially only one parameter $\Delta / \lambda$ which characterizes both the anisotropy as well as the $g$ values for the magnetic ion [22]. The secular equations for the energies were solved numerically in terms of $\Delta^{\prime} / \lambda$. The energies as a function of the positive and negative values of $\Delta^{\prime} / \lambda$ are shown in figure $2 .\left(\Delta^{\prime}=3 \Delta\right)$.

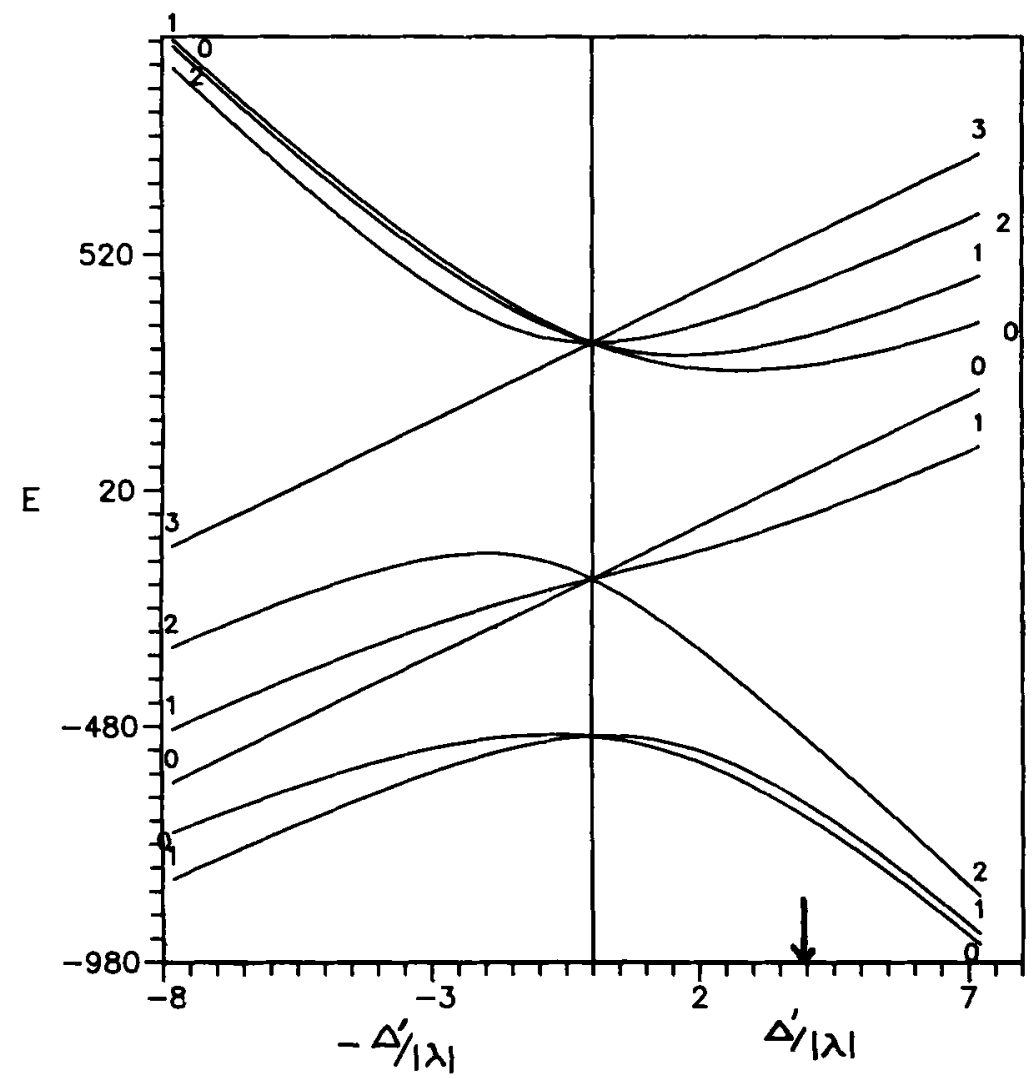

Figure 2. The splitting of the ground term of the high spin ferrous ion in a trigonal field. The energy in units of kelvin is given along the $y$-axis. The $M_{j}$ values are marked against the corresponding energy state. The arrow indicates the value of $\Delta^{\prime} /|\lambda|$ for FePS 3 as obtained from the analysis of the high temperature susceptibility. 


\section{Nirmala Chandrasekharan and Sukumaran Vasudevan}

The above Hamiltonian may be simplified under two extreme conditions i) $\lambda \gg \Delta$ or ii) $\Delta \gg \lambda$. In the first instance one need consider only the splitting of the ${ }^{5} T_{2 q}$ state by the spin-orbit coupling into the three levels ${ }^{5} T_{21},{ }^{5} T_{22},{ }^{5} T_{23}$. For FePS ${ }_{3}$ this is clearly not the situation since it would not account (see figure 4) for the experimentally observed large differences between $\chi_{\|}$and $\chi_{\perp}$. In situations where $\Delta \gg \lambda$ one first considers the splitting of the ${ }^{5} T_{2 g}$ state by the low symmetry trigonal field into a doublet $\left({ }^{5} E_{\theta}\right)$ with $L_{z}= \pm 1$ and the singlet $\left({ }^{5} A_{1 g}\right), L_{z}=0$. The effect of the spin-orbital coupling is considered only within the ground state manifold (either the ${ }^{5} E_{g}$ or ${ }^{5} A_{1 g}$ ). In an earlier mean field analysis of the susceptibility of $\mathrm{FePS}_{3}$ [5], the above assumption $(\Delta \gg \lambda)$ had been made and only those states arising from the effect of spin-orbit coupling on the ${ }^{5} E_{g}\left(L_{z}^{\prime}\right)$ state were considered in the calculation of the susceptibility. In the light of the optical absorption data of $\mathrm{FePS}_{3}[7]$ as well as the vibration spectra $[10]$ the above assumption seems unjustified. Both experiments showed that in $\mathrm{FePS}_{3}$ the iron atoms are in a highly ionic environment. Consequently the spin-orbit coupling constant is likely to be close to that of the free ion value of $-100 \mathrm{~cm}^{-1}$ [22]. If $\Delta$ was indeed much greater than $\lambda$ the effect of the trigonal distortion field should have been observed in the optical spectra. This was not so and the simplification $\Delta \gg \lambda$ is unlikely to hold.

Since the Hamiltonian cannot be simplified for $\mathrm{FePS}_{3}$ it implies that the entire 15 level structure of the ${ }^{5} T_{2}$ state would have to be included in the evaluation of any ground state thermodynamic property.

Although the $\mathrm{Fe}^{2+}$ ions are orbitally degenerate, it is assumed that the exchange is isotropic and between real spins, so that ith ion spin Hamiltonian may be written as

$$
\mathscr{H}=\Delta\left(L_{z}^{\prime 2}-2\right)-|\lambda| \mathbf{L}^{\prime} \cdot \mathbf{S}-\sum_{j} J_{i j} \mathbf{S}_{i} \cdot \mathbf{S}_{j}
$$

and the effective CEF Hamiltonian in the paramagnetic phase and in the absence of a magnetic field is

$$
\mathscr{H}_{i}^{0} \mathrm{eff}=\Delta\left(L_{z}^{\prime 2}-2\right)-|\lambda| \mathbf{L}^{\prime} \cdot \mathrm{S}-\sum_{j} J_{i j}\left(\alpha_{\|}-\alpha_{\perp}\right) S_{i z}^{2}
$$

Here $\|$ refers to the external field applied along the trigonal axis. The summation extends to the next nearest neighbour. This is necessary if the in-plane antiferromagnetic ordered structure for $\mathrm{FePS}_{3}$ [23] is to be realized.

The correlation parameters were obtained from the solution of (9). The Brillouin zone average in the calculation of $\alpha$ was computed using the special $k$ points for the $2 D$ hexagonal lattice [20]. It may be seen that the equation for $\alpha_{\|}$and $\alpha_{\perp}$ are not independent and were solved numerically. The variation of $\alpha$ 's as a function of temperature is shown in figure 3 . The static susceptibilities were calculated using (10). The exchange constants $J_{n n}, J_{n n n}$ as well as $\Delta$ and $\lambda$ were obtained by a least square fit of the CEF susceptibility to the experimental data. The best fit is shown as the solid line in figure 4 obtained for $J_{n n} / k=27.7 \mathrm{~K} ; J_{n n n} / k=-2.3 \mathrm{~K} ; \Delta / k=215.5 \mathrm{~K}$; $|\lambda| / k=166.5 \mathrm{~K}$.

The fit is reasonably good and the $\lambda$ value close to that of the free ion value for $\mathrm{Fe}^{2+}$. This is in agreement with other experimental observations that in FePS 3 the $\mathrm{Fe}-\mathrm{S}$ linkage is ionic. The results of the fit also justifies the retaining of the entire 15 level structure of the ${ }^{5} T_{2 g}$ state without any simplification in the calculation of the susceptibilities, since the value of $\Delta$ and $|\lambda|$ obtained are comparable $(\Delta /|\lambda|=1 \cdot 3)$. 


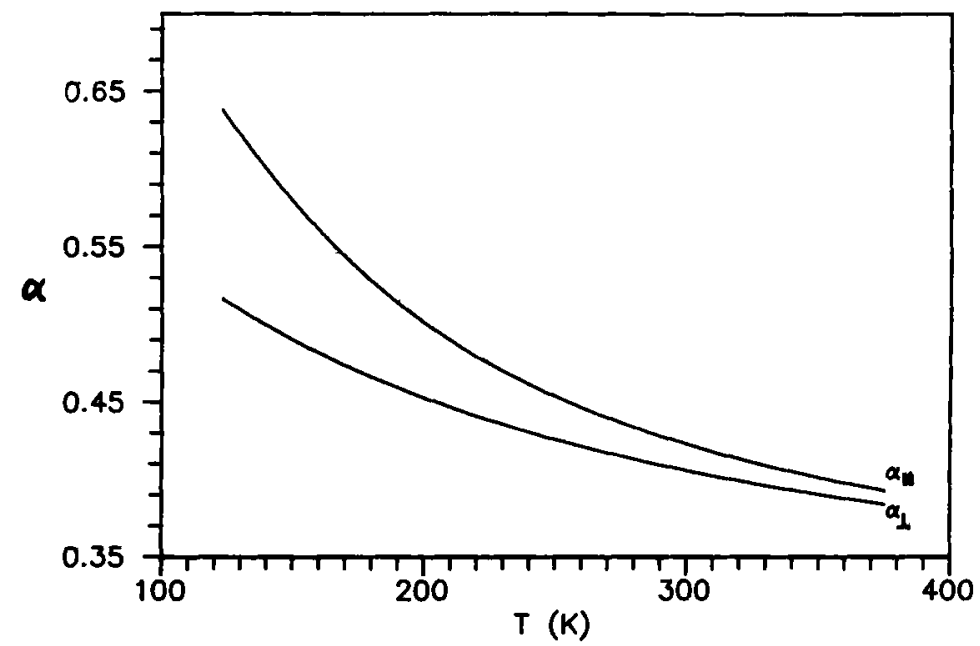

Figure 3. The temperature dependence of the correlation parameter $\alpha_{\|}$and $\alpha_{\perp}$ calculated from the self-consistent Eq. (9) for FePS .

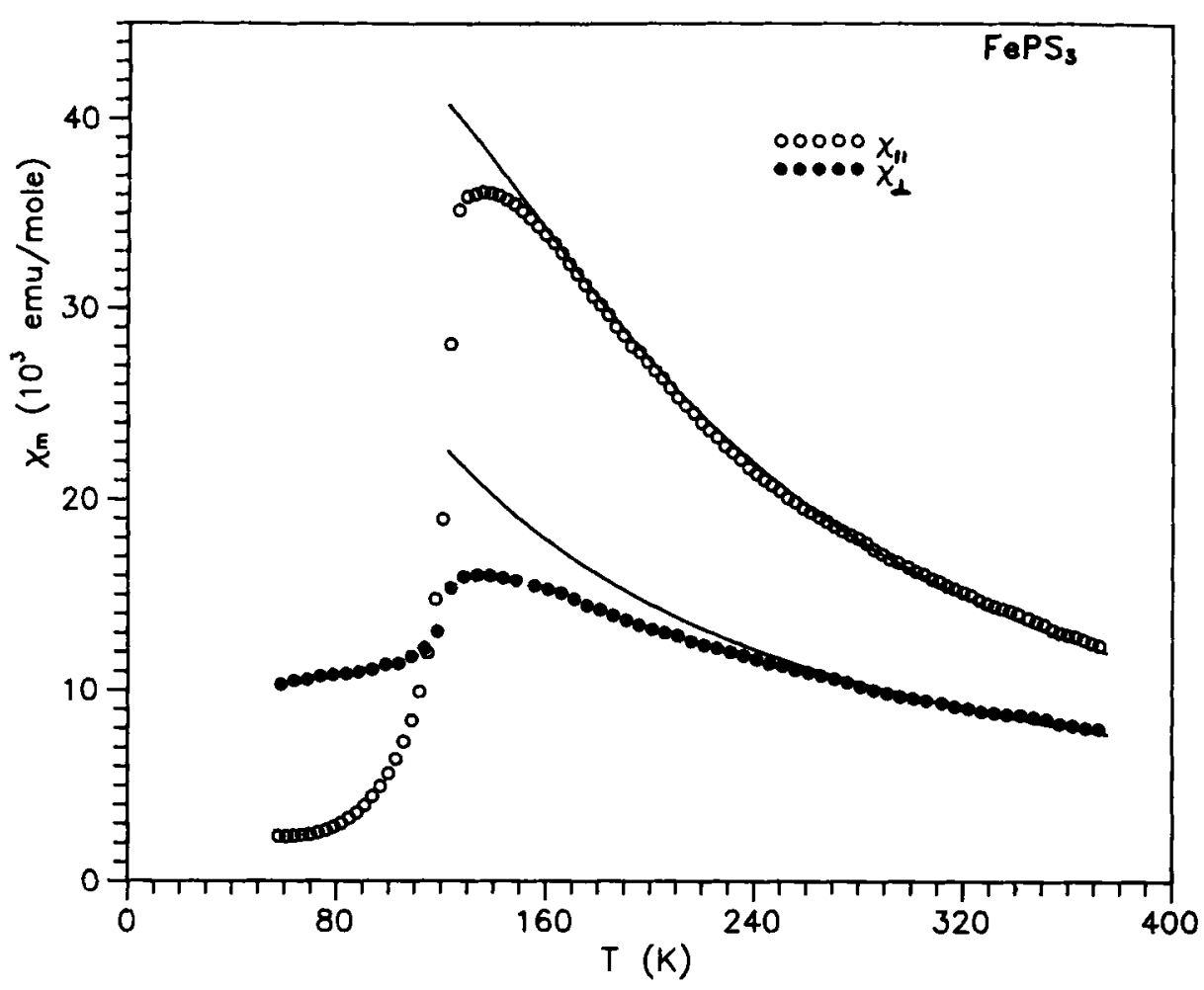

Figure 4. The anisotropic magnetic susceptibilities of FePS ${ }_{3}$ parallel $\left(\chi_{\|}\right)$and perpendicular $\left(\chi_{\perp}\right)$ to the trigonal axis, as a function of temperature. The solid lines are the best least squares CEF fit of the anisotropic susceptibility expression of eq. (10) to the experimental susceptibilities. The values of the microscopic parameters are $J_{n n} / k=27.7 \mathrm{~K} ; J_{n n n} / K=-2.3 \mathrm{~K} ;|\lambda| / k=166.5 \mathrm{~K}$ and $\Delta^{\prime} / k=215.5 \mathrm{~K}$. 


\section{Nirmala Chandrasekharan and Sukumaran Vasudevan}

The results of the present CEF calculation are a considerable improvement on the earlier MFA analysis of Joy et al [5]. The earlier MFA analysis, apart from having assumed that $\Delta \gg \lambda$ could be fitted to the experimental data only by forcing an unphysical situation, in which the exchange constants $J$ had opposite signs for $\chi_{\|}$ and $\chi_{1}$. Yet another merit of the CEF model, is its accurate prediction of the value of $T_{\mathrm{N}}$. Substituting the values of $J$ 's and $\alpha$ from the fit in (11) gave a $T_{\mathrm{N}}$ value of $122 \mathrm{~K}$. This is remarkably close to the experimental value of $123 \mathrm{~K}$.

\section{Conclusions}

The anisotropic magnetic susceptibility of the $2 D$ orbitally unquenched Ising antiferromagnet $\mathrm{FePS}_{3}$ has been analyzed in the CEF approximation, developed by Lines. The formalism introduces spin correlations in the simplest possible manner while at the same time accounting for the excited states of the orbitally unquenched transition metal ion, the energies of which are comparable to exchange and thermal energies. In $\mathrm{FePS}_{3}$ the degeneracy of the ${ }^{5} \Gamma_{2}$ state is completely lifted by the combination of trigonal distortion of the $\mathrm{FeS}_{6}$ octahedra, spin-orbit coupling and Zeeman splitting. In the calculation of the susceptibilities in the CEF approximation the entire 15 level structure of the ${ }^{5} \Gamma_{2}$ state was retained. Good agreement with experiment were obtained for $\Delta / k=215.5 \mathrm{~K} ;|\lambda| / k=166.5 \mathrm{~K} ; J_{n n} / k=27.7 \mathrm{~K}$; and $J_{n n n} / k=-2.3 \mathrm{~K}$. Using these values of the crystal field and exchange parameters the CEF predicts a $T_{\mathrm{N}}=122 \mathrm{~K}$ for $\mathrm{FePS}_{3}$ which is remarkably close to the observed value of $T_{\mathrm{N}}$. The accuracy of the CEF approximation was also ascertained, by comparison of the calculated susceptibilities in CEF with the experimental susceptibility for the isotropic Heisenberg layered antiferromagnet $\mathrm{MnPS}_{3}$ for which the high temperature series expansion susceptibility is available. The comparison also establishes the effectiveness of the special $k$ point scheme in providing Brillouin zone averages in the computation of susceptibilities in the CEF approximation.

The results of the present analysis show that the CEF approximation provides a simple and elegant way to analyze the susceptibilities of exchange coupled systems in which the individual ion possesses a complicated level structure. In a subsequent paper, we shall show how the CEF approximation can be extended to more complicated multicomponent systems, like the solid solutions of the transition metal thiophosphates of the formula $M_{1-x}^{\prime} M_{x} P S_{3}\left(M^{\prime}, M=\mathrm{Mn}, \mathrm{Fe}, \mathrm{Ni}\right)$ where $M$ and $M^{\prime}$ have different energy level structures.

\section{References}

[1] W Klingen, R Otto and H Z Hahn, Z. Anorg. Allg. Chem. 396, 271 (1973)

[2] R Brec, Solid State Ionics 22, 3 (1986)

[3] L J De Jongh and A R Miedema, Adv. Phys. 23, 1 (1974)

[4] G Le Flem, R Brec, G Ouvrard, A Louisy and P Segransen, J. Phys. Chem. Solids 43, 455 (1982)

[5] P A Joy and S Vasudevan, Phys. Rev. B46, 5425 (1992)

[6] P A Joy and S Vasudevan, J. Phys. Chem. Solids 54, 343 (1993)

[7] P A Joy and S Vasudevan, Phys. Rev. B46, 5134 (1992)

[8] S Whittingham and A Jacobson (ed.) Intercalation chemistry (Academic Press, New York, 1982)

[9] P A Joy and S Vasudevan, J. Am. Chem. Soc. 114, 7792 (1992)

[10] P A Joy and S Vasudevan, J. Chem. Phys. 99, 4411 (1993) 
[11] R Clement, L Lomas and J P Audiere, Chem. Mater. 2, 641 (1990)

[12] P A Joy and S Vasudevan, Chem. Mater. 5, 1182 (1993)

[13] M E Lines, Phys. Rev. B9, 3927 (1974)

[14] M E Lines, Phys. Rev. B9, 3766 (1975)

[15] M E Lines and M Eibschütz, Phys. Rev. B11, 4853 (1975)

[16] M Eibschütz, M E Lines and L C Sherwood, Phys. Rev. B11, 4595 (1975)

[17] T Oguchi, Prog. Theor. Phys. (Kyoto) 13, 148 (1955)

[18] J S Smart, Effective field theories of magnetism (W B Saunders Co., 1966)

[19] D J Chadi and M L Cohen, Phys. Rev. B8, 5947 (1973)

[20] S L Cunningham, Phys. Rev. B10, 4988 (1974) The wavevectors (in units of $\pi / a$ ) for the 2D Hexagonal lattice along with the weightage factors are $(2 / 9,2 / 9 \mathrm{t}), 1 / 9 ;(4 / 9,4 / 9 \mathrm{t}), 1 / 9$; $(8 / 9,8 / 9 t), 1 / 9 ;(2 / 3,2 / 9 t), 2 / 9 ;(8 / 9,4 / 9 t), 2 / 9 ;(10 / 9,2 / 9 t), 2 / 9 . t=\sqrt{3}$

[21] G S Rushbrook and P J Wood, Mol. Phys. 1, 257 (1958)

[22] J S Griffith, The theory of transition-metal ions (Cambridge University Press, Cambridge, 1961)

[23] K Kurosawa, S Saito and Y Yamaguchi, J. Phys. Soc. Jpn. 52, 3919 (1983) 